\title{
Electron-electron relaxation in two-dimensional impure superconductors
}

\author{
Michael Reizer \\ 5614 Naiche Rd. Columbus $\mathrm{OH} 43213$
}

(April 25, 2018)

\begin{abstract}
The electron-electron relaxation in impure two-dimensional superconductors is studied. All channels of the electron-electron interaction classified in the Nambu representation are taken into account. It is shown that the recombination relaxation rate originates from quasipartical processes associated with fluctuations of the electron density and the phase of the order parameter. At low temperatures the recombination relaxation rate has a double exponential temperature dependence. The scattering relaxation rate at low temperatures has a power law temperature dependence due to contributions from gapless collective excitations, the phase modes. Two-layer superconductornormal metal system is also considered. It is shown that the recombination relaxation rate in the superconducting layer has a single exponential factor at low temperatures in comparison with a one layer superconducting system. This increase in the recombination relaxation rate originates from the interlayer Coulomb interaction and may be used in constructing of superconducting radiation detectors.
\end{abstract}

PACS: 73.50.Bk, 73.50.Dn. 


\section{INTRODUCTION}

The electron-electron energy-relaxation time determines a number of parameters of nonequilibrium superconductors such as the relaxation times for the amplitude and the phase of the order parameter [1]. It is also important for nonequilibrium superconducting radiation detectors based on the resistive and inductive responses. The electron-electron relaxation time is responsible for the quasiparticle multiplication coefficient which in turn determines the responsivity and detectivity of the detector and its noise characteristics [2].

The energy relaxation time also serves as a pair-breaking parameter in the superconducting density of states which is measured in the tunneling experiment [3]. Recently the energy relaxation time was measured in cuprate superconductors by studying an electronic instability at high vortex velocities in the mixed state [4].

The electron-electron relaxation time of clean superconductors was calculated for the three-dimensional case in Ref. [5] and for the two-dimensional case in Ref. [6]. In the last work all channels of the electron-electron interaction not only the Coulomb interaction was taken into account by using the matrix classification of the interaction channels developed earlier in Refs. [7] and [8]. The importance of considering all channels of interaction was realized long ago for the problem of gauge invariance in superconductors [9]. It was also emphasized in Ref. [8] that interference between different channels of interaction cancels divergences in the interaction correction to the superconducting order parameter.

It is known that in normal impure and low dimensional metals the diffusive motion of electrons leads to enhancement of the electron-electron relaxation [10, [11]. The purpose of the present paper is to calculate the electron-electron relaxation time in impure twodimensional superconductors using the formalism of Refs. [7] and [8]. Earlier attempt to study electron-electron relaxation in impure two-dimensional superconductors [12] took into account only the Coulomb electron-electron interaction and therefore ignored the other relevant interaction channels. As we will show in the present paper including all channels of the interaction is very important for the electron-electron relaxation, and leads to results 
qualitatively different from that of Ref. [12]. Another difference is that we found important contributions to the scattering relaxation time from gapless collective excitations which where mised in Ref. [12]. Note also that we derived the quasiparticle energy relaxation time from the quantum kinetic equation, not as an imaginary part of the self-energy as in Ref. [12].

Then we study the superconductor-normal metal two-layer system. Such a system was already studied in Ref. [6] for a clean system. We consider a disordered case in the present work and show that recombination relaxation rate is strongly enhanced due to inter-layer electron-electron interaction.

\section{ELECTRON-ELECTRON INTERACTION IN MATRIX FORMALISM}

We use the Keldysh diagram technique for nonequilibrium processes in which the electron Green's functions, along with the electron-electron interaction potential, the electron self energy and the polarization operator are represented by supermatrices

$$
\begin{array}{cc}
(\hat{G})=\left(\begin{array}{cc}
0 & \hat{G}^{A} \\
\hat{G}^{R} & \hat{G}^{C}
\end{array}\right), \quad(\hat{V})=\left(\begin{array}{cc}
0 & \hat{V}^{A} \\
\hat{V}^{R} & \hat{V}^{C}
\end{array}\right), \\
(\hat{\Sigma})=\left(\begin{array}{cc}
\hat{\Sigma}^{C} & \hat{\Sigma}^{R} \\
\hat{\Sigma}^{A} & 0
\end{array}\right), \quad(\hat{\Pi})=\left(\begin{array}{cc}
\hat{\Pi}^{C} & \hat{\Pi}^{R} \\
\hat{\Pi}^{A} & 0
\end{array}\right) .
\end{array}
$$

The matrix electron Green's function in an impure superconductor in the Nambu representation has the form

$$
\begin{aligned}
\hat{G}^{R}(P)=\left[\hat{G}^{A}(P)\right]^{*} & =\frac{-\xi_{p} \hat{\tau}_{3}-\epsilon^{R} \hat{\tau}_{0}+\Delta^{R} \hat{\tau}_{1}}{\xi_{p}^{2}-\left(E^{R}\right)^{2}}, \\
P & =(\mathbf{p}, \epsilon), \quad \xi_{p}=\frac{p^{2}-p_{F}^{2}}{2 m},
\end{aligned}
$$

where $\hat{\tau}_{i}$ are the Pauli matrices, $m$ is the electron mass, and

$$
\begin{aligned}
\epsilon^{R}=\epsilon\left(1+\frac{i}{2 \tau \xi_{\epsilon}}\right), \quad \Delta^{R} & =\Delta\left(1+\frac{i}{2 \tau \xi_{\epsilon}}\right), \\
\left(E^{R}\right) & =\left(\epsilon^{R}\right)^{2}+\left(\Delta^{R}\right)^{2},
\end{aligned}
$$


where $\Delta$ is the energy gap, $\tau$ is the electron-impurity relaxation time, and

$$
\xi_{\epsilon}=\left(\epsilon^{2}-\Delta^{2}\right)^{1 / 2} \operatorname{sgn}(\epsilon), \quad|\epsilon|>\Delta .
$$

In a spatially uniform system the kinetic components $\hat{G}^{C}$ and $\hat{\Sigma}^{C}$ are satisfied the equations

$$
\begin{aligned}
\hat{G}^{C}(P) & =S(\epsilon)\left[\hat{G}^{A}(P)-\hat{G}^{R}(P)\right], \\
\hat{\Sigma}(P) & =S(\epsilon)\left[\hat{\Sigma}^{A}(P)-\hat{\Sigma}^{R}(P)\right],
\end{aligned}
$$

where $S(\epsilon)=-\tanh (\epsilon / 2 T)=2 n(\epsilon)-1$, and $n(\epsilon)$ is the Fermi distribution function. Similar relations hold for matrix interaction potentials and polarization operators:

$$
\begin{gathered}
\hat{V}^{C}(Q)=(2 N(\omega)+1)\left[\hat{V}^{R}(Q)-\hat{V}^{A}(Q)\right], \\
\hat{\Pi}^{C}(Q)=(2 N(\omega)+1)\left[\hat{\Pi}^{R}(Q)-\hat{\Pi}^{A}(Q)\right],
\end{gathered}
$$

where $Q=(\mathbf{q}, \omega)$ and $N(\omega)$ is the Bose distribution function.

For averaging over impurity position it is convenient to introduce the following expressions

$$
\begin{array}{r}
\eta_{i}^{A A}=\frac{1}{\pi \nu \tau}<\hat{\tau}_{3} \hat{G}^{A}(P) \hat{\tau}_{i} \hat{G}^{A}(P+Q) \hat{\tau}_{3}>, \\
\eta_{i}^{A R}=\frac{1}{\pi \nu \tau}<\hat{\tau}_{3} \hat{G}^{A}(P) \hat{\tau}_{i} \hat{G}^{R}(P+Q) \hat{\tau}_{3}>, \\
<\ldots>=\int \frac{d^{2} p}{(2 \pi)^{2}},
\end{array}
$$

where $\nu$ is the two-spin electron density of states. Calculations give e.g.

$$
\begin{aligned}
& \eta_{0}^{A R}=\frac{\zeta}{2}\left[\left(1+A_{+}\right) \hat{\tau}_{0}+B_{+} \hat{\tau}_{1}\right], \\
& \eta_{3}^{A A}=\frac{\zeta_{+}}{2}\left[(1-A) \hat{\tau}_{3}+B i \hat{\tau}_{2}\right],
\end{aligned}
$$

where

$$
\begin{array}{r}
A=\frac{\epsilon(\epsilon+\omega)-\Delta^{2}}{\xi_{\epsilon} \xi_{\epsilon+\omega}}, \quad A_{+}=\frac{\epsilon(\epsilon+\omega)+\Delta^{2}}{\xi_{\epsilon} \xi_{\epsilon+\omega}}, \\
B=\frac{(\epsilon+\omega) \Delta-\epsilon \Delta}{\xi_{\epsilon} \xi_{\epsilon+\omega}}=\frac{\omega \Delta}{\xi_{\epsilon} \xi_{\epsilon+\omega}}, \\
B_{+}=\frac{(\epsilon+\omega) \Delta+\epsilon \Delta}{\xi_{\epsilon} \xi_{\epsilon+\omega}}=\frac{(2 \epsilon+\omega) \Delta}{\xi_{\epsilon} \xi_{\epsilon+\omega}},
\end{array}
$$




$$
\begin{gathered}
\zeta=1+i\left(\xi_{\epsilon+\omega}-\xi_{\epsilon}\right) \tau-D q^{2} \tau, \\
\zeta_{+}=1-i\left(\xi_{\epsilon+\omega}+\xi_{\epsilon}\right) \tau-D q^{2} \tau .
\end{gathered}
$$

Values of $\eta_{i}$ for different matrices $\hat{\tau}_{i}$ are presented in Tab. 1. Note also that the following identities hold: $A^{2}-B^{2}=1$ and $A_{+}^{2}-B_{+}^{2}=-1$.

Treating the electron-electron interaction in superconductors we use the matrix formalism developed in Refs. 7 and 8. The bare vertices for the electron-electron interaction are classified in terms of the Pauli matrices. Physical meaning of the corresponding operators $\hat{O}_{i}=\Psi^{\dagger} \hat{\tau}_{i} \Psi$ is the following. Matrix $\hat{\tau}_{1}$ corresponds to the order parameter amplitude $\Delta$, matrix $\hat{\tau}_{2}$ corresponds to the order parameter phase $\phi$, matrix $\hat{\tau}_{3}$ corresponds to the electron density, and the vector matrix $\mathbf{k} \hat{\tau}_{0}$ corresponds to the electric current, the later will not be considered in the present paper(see Ref. 5). Note also that each impurity vertex carries the matrix $\hat{\tau}_{3}$.

Therefore each interaction vertex operates in both Keldysh and Nambu spaces and has the form $\hat{\gamma}_{m n}^{k}\left(\hat{\tau}_{i}\right)=\hat{\gamma}\left(\hat{\tau}_{i}\right) K_{m n}^{k}$, where $\hat{\tau}_{i}$ indicates the component in the Nambu space and tensor $K_{m n}^{k}$ stands for the Keldysh space, $k$ is a boson index and $m$ and $n$ are the electron indices. In the representation corresponding to Eq. 1 the nonzero components of tensor $K_{m n}^{k}$ are

$$
K_{22}^{1}=K_{11}^{1}=K_{12}^{2}=K_{21}^{2}=\frac{1}{\sqrt{2}} .
$$

We will omit coefficient $1 / \sqrt{2}$ in intermediate equations for the impurity renormalized vertices and restore it in final equations for the polarization operators and the electron self energies.

Impurity averaging leads to the ladder equation for the scalar vertex $\hat{\Gamma}\left(\hat{\tau}_{i}\right)$ shown in Fig. 1. Such an equation should be written for each bare matrix $\hat{\tau}_{i}$ in the Nambu space. The solution of these equations for the vertex $\hat{\Gamma}\left(\hat{\tau}_{i}\right)$ in the Keldysh-Nambu space is obtained following Ref. 13. We start with the equation for the vertex $\hat{\Gamma}_{22}^{1}\left(\hat{\tau}_{3}\right)$,

$$
<\hat{\tau}_{3} \hat{G}^{A}(P) \hat{\Gamma}_{22}^{1}\left(\hat{\tau}_{3}\right) \hat{G}^{A}(P+Q) \hat{\tau}_{3}>-\hat{\Gamma}_{22}^{1}\left(\hat{\tau}_{3}\right)=-\hat{\tau}_{3}
$$


The solution of this equation is

$$
\hat{\Gamma}_{22}^{1}\left(\hat{\tau}_{3}\right)=-\frac{\zeta}{2} \frac{B}{1-\zeta} i \hat{\tau}_{2}+\left(1+\frac{\zeta}{2} \frac{1+A}{1-\zeta}\right) \hat{\tau}_{3}
$$

Note that renormalized vertex $\hat{\Gamma}_{22}^{1}\left(\hat{\tau}_{3}\right)$ has components proportional not only to to matrix $\hat{\tau}_{3}$ but also to matrix $\hat{\tau}_{2}$. The other vertices $\hat{\Gamma}_{22}^{1}\left(\hat{\tau}_{i}\right)$ and $\hat{\Gamma}_{21}^{2}\left(\hat{\tau}_{i}\right)$ are presented in Tab. 2 . The vertices with the other Keldysh indices are obtained from the equations

$$
\begin{array}{r}
\hat{\Gamma}_{12}^{2}\left(\hat{\tau}_{i}\right)=\left(\hat{\Gamma}_{21}^{2}\left(\hat{\tau}_{i}\right)\right)^{*}, \quad \hat{\Gamma}_{12}^{1}\left(\hat{\tau}_{i}\right)=S(\epsilon)\left[\hat{\Gamma}_{22}^{1}\left(\hat{\tau}_{i}\right)-\hat{\Gamma}_{12}^{2}\left(\hat{\tau}_{i}\right)\right] \\
\hat{\Gamma}_{21}^{1}\left(\hat{\tau}_{i}\right)=-S(\epsilon+\omega)\left[\hat{\Gamma}_{22}^{1}\left(\hat{\tau}_{i}\right)-\hat{\Gamma}_{21}^{2}\left(\hat{\tau}_{i}\right)\right], \\
\hat{\Gamma}_{11}^{2}\left(\hat{\tau}_{i}\right)=S(\epsilon) \hat{\Gamma}_{21}^{2}\left(\hat{\tau}_{i}\right)-S(\epsilon+\omega) \hat{\Gamma}_{12}^{2}\left(\hat{\tau}_{i}\right) \\
-[S(\epsilon)-S(\epsilon+\omega)]\left(\hat{\Gamma}_{22}^{1}\left(\hat{\tau}_{i}\right)\right)^{*}
\end{array}
$$

Note also that index structure of the renormalized vertices in the Keldysh space is different from the index structure of the bare vertex described by Eq. (11). It is important that the complex conjugate in Eq. (14) operates only in the Keldysh space, thus any $i$ in Tab. 2 originating from $\hat{\tau}_{i}$ matrix algebra are not affected by the complex conjugate, e. g. (compare with Eq. (13))

$$
\left(\hat{\Gamma}_{22}^{1}\left(\hat{\tau}_{3}\right)\right)^{*}=-\frac{\zeta^{*}}{2} \frac{B}{1-\zeta^{*}} i \hat{\tau}_{2}+\left(1+\frac{\zeta^{*}}{2} \frac{1+A}{1-\zeta^{*}}\right) \hat{\tau}_{3}
$$

The effective screened electron-electron interaction in a superconductor according to Refs. 6-8 is shown in Fig. 1. The solution of this equation in $3 \times 3$ matrix form is

$$
\hat{V}=\left(\begin{array}{ccc}
-\left(2 / \lambda+\Pi_{11}\right)^{-1} & 0 & 0 \\
0 & {\left[\left(V_{0}\right)^{-1}-\Pi_{33}\right] / \mathcal{D}} & \Pi_{23} / \mathcal{D} \\
0 & \Pi_{32} / \mathcal{D} & -\left(2 / \lambda+\Pi_{22}\right) / \mathcal{D}
\end{array}\right),
$$

where

$$
\mathcal{D}=-\left(2 / \lambda+\Pi_{22}\right)\left[\left(V_{0}\right)^{-1}-\Pi_{33}\right]-\Pi_{23} \Pi_{32},
$$

and $\lambda$ is the BCS coupling constant $(\lambda>0), V_{0}=2 \pi e^{2} / q$ is the nonscreened two-dimensional Coulomb potential. 
The polarization operators renormalized by impurities are expressed through the vertex $\hat{\Gamma}$ by the equation

$$
\Pi_{i j}^{A}(Q)=-\frac{i}{2} \pi \nu \tau \int \frac{d \epsilon}{2 \pi} \operatorname{Sp}\left(\hat{\tau}_{3} \hat{\tau}_{j} \hat{\tau}_{3} \Gamma_{11}^{2}\left(\hat{\tau}_{i}\right)\right)
$$

Using Eq. (14) for $\Gamma_{11}^{2}\left(\hat{\tau}_{i}\right)$ and Tab. 2 we find the polarization operators,

$$
\begin{gathered}
\Pi_{11}^{A}(Q)=\frac{i \nu \tau}{4} \int d \epsilon\left(\left(1-A_{+}\right) \frac{S(\epsilon+\omega)-S(\epsilon)}{1-\zeta^{*}}-\left(1+A_{+}\right)\left[\frac{S(\epsilon+\omega)}{1-\zeta_{+}^{*}}-\frac{S(\epsilon)}{1-\zeta_{+}}\right]\right), \\
\Pi_{22}^{A}(Q)=\frac{i \nu \tau}{4} \int d \epsilon\left((1-A) \frac{S(\epsilon+\omega)-S(\epsilon)}{1-\zeta^{*}}-(1+A)\left[\frac{S(\epsilon+\omega)}{1-\zeta_{+}^{*}}-\frac{S(\epsilon)}{1-\zeta_{+}}\right]\right), \\
\Pi_{33}^{A}(Q)=-\nu-\frac{i \nu \tau}{4} \int d \epsilon\left((1+A) \frac{S(\epsilon+\omega)-S(\epsilon)}{1-\zeta^{*}}-(1-A)\left[\frac{S(\epsilon+\omega)}{1-\zeta_{+}^{*}}-\frac{S(\epsilon)}{1-\zeta_{+}}\right]\right), \\
\Pi_{32}^{A}(Q)=-\Pi_{23}^{A}(Q)=-\frac{\nu \tau}{4} \int d \epsilon B\left(\frac{S(\epsilon)-S(\epsilon+\omega)}{1-\zeta^{*}}-\frac{S(\epsilon+\omega)}{1-\zeta_{+}^{*}}+\frac{S(\epsilon)}{1-\zeta_{+}}\right) .
\end{gathered}
$$

To calculate the electron relaxation we need the imaginary part of the potentials (the polarization operators) in the quasiparticle representation. Making a transformation from the electronic representation to the quasiparticle representation we use Eq. (4). As a result we separate out the processes of scattering and recombination of quasiparticles in Eqs. (18)(21). For the imaginary part of the polarization operators we have

$$
\begin{gathered}
\operatorname{Im}_{i i}^{A}(Q)_{s c a t t}=\frac{\nu}{2} \int_{\Delta}^{\infty} d \epsilon[S(\epsilon+\omega)-S(\epsilon)] C_{i i}(q, \epsilon, \omega), \\
\operatorname{Im}_{i i}^{A}(Q)_{\text {recom }}=\frac{\nu}{4} \Theta(\omega-2 \Delta) \int_{\Delta}^{\omega-\Delta} d \epsilon[S(\epsilon-\omega)-S(\epsilon)] C_{i i}(q, \epsilon,-\omega),
\end{gathered}
$$

where $C_{i i}$ are

$$
\begin{aligned}
C_{11}(q, \epsilon, \omega) & =\left(1-\frac{\epsilon(\epsilon+\omega)+\Delta^{2}}{\left|\xi_{\epsilon}\right|\left|\xi_{\epsilon+\omega}\right|}\right) \frac{D q^{2}}{\left(\left|\xi_{\epsilon+\omega}\right|-\left|\xi_{\epsilon}\right|\right)^{2}+\left(D q^{2}\right)^{2}} \\
& -\left(1+\frac{\epsilon(\epsilon+\omega)+\Delta^{2}}{\left|\xi_{\epsilon}\right|\left|\xi_{\epsilon+\omega}\right|}\right) \frac{D q^{2}}{\left(\left|\xi_{\epsilon+\omega}\right|+\left|\xi_{\epsilon}\right|\right)^{2}+\left(D q^{2}\right)^{2}}, \\
C_{22}(q, \epsilon, \omega) & =\left(1-\frac{\epsilon(\epsilon+\omega)-\Delta^{2}}{\left|\xi_{\epsilon}\right|\left|\xi_{\epsilon+\omega}\right|}\right) \frac{D q^{2}}{\left(\left|\xi_{\epsilon+\omega}\right|-\left|\xi_{\epsilon}\right|\right)^{2}+\left(D q^{2}\right)^{2}}
\end{aligned}
$$




$$
\begin{aligned}
- & \left(1+\frac{\epsilon(\epsilon+\omega)-\Delta^{2}}{\left|\xi_{\epsilon}\right|\left|\xi_{\epsilon+\omega}\right|}\right) \frac{D q^{2}}{\left(\left|\xi_{\epsilon+\omega}\right|+\left|\xi_{\epsilon}\right|\right)^{2}+\left(D q^{2}\right)^{2}}, \\
C_{33}(q, \epsilon, \omega)= & -\left(1+\frac{\epsilon(\epsilon+\omega)-\Delta^{2}}{\left|\xi_{\epsilon}\right|\left|\xi_{\epsilon+\omega}\right|}\right) \frac{D q^{2}}{\left(\left|\xi_{\epsilon+\omega}\right|-\left|\xi_{\epsilon}\right|\right)^{2}+\left(D q^{2}\right)^{2}} \\
& +\left(1-\frac{\epsilon(\epsilon+\omega)-\Delta^{2}}{\left|\xi_{\epsilon}\right|\left|\xi_{\epsilon+\omega}\right|}\right) \frac{D q^{2}}{\left(\left|\xi_{\epsilon+\omega}\right|+\left|\xi_{\epsilon}\right|\right)^{2}+\left(D q^{2}\right)^{2}} .
\end{aligned}
$$

For the off-diagonal polarization operator we have

$$
\begin{gathered}
\Pi_{32}^{A}(Q)_{s c a t t}=-\frac{\nu}{2} \Delta \omega \int_{\Delta}^{\infty} \frac{d \epsilon}{\left|\xi_{\epsilon}\right|\left|\xi_{\epsilon+\omega}\right|}\left[\frac{(S(\epsilon)-S(\epsilon+\omega))}{i\left|\xi_{\epsilon+\omega}\right|-i\left|\xi_{\epsilon}\right|+D q^{2}}\right. \\
\left.+\frac{S(\epsilon)}{i\left|\xi_{\epsilon+\omega}\right|+i\left|\xi_{\epsilon}\right|+D q^{2}}-\frac{S(\epsilon+\omega)}{-i\left|\xi_{\epsilon+\omega}\right|-i\left|\xi_{\epsilon}\right|+D q^{2}}\right], \\
\Pi_{32}^{A}(Q)_{r e c}=-\Theta(\omega-2 \Delta) \frac{\nu}{2} \Delta \omega \int_{\Delta}^{\omega-\Delta} \frac{d \epsilon}{\left|\xi_{\epsilon}\right|\left|\xi_{\epsilon-\omega}\right|}\left[\frac{S(\epsilon)-S(\epsilon-\omega)}{i\left|\xi_{\epsilon-\omega}\right|-i\left|\xi_{\epsilon}\right|+D q^{2}}\right. \\
\left.+\frac{S(\epsilon)}{i\left|\xi_{\epsilon-\omega}\right|+i\left|\xi_{\epsilon}\right|+D q^{2}}-\frac{S(\epsilon-\omega)}{-i\left|\xi_{\epsilon-\omega}\right|-i\left|\xi_{\epsilon}\right|+D q^{2}}\right] .
\end{gathered}
$$

As we will see in the next chapter, calculating the electron relaxation time we need the imaginary part of the propagators $\operatorname{Im}\left(V_{i i}^{A}(Q)\right)$. The imaginary part of the propagators may originate from the poles of the propagators which correspond to collective excitations or from the imaginary part of the polarization operators $\operatorname{Im}\left(\Pi_{i i}^{A}(Q)\right)$, which correspond to real processes of scattering and recombination of quasiparticles. We will restrict our calculations to low temperatures $T<<\Delta$, where large frequencies $\omega-2 \Delta<<T$, are important for both recombination and scattering processes and small frequencies $\omega<<T<<\Delta$ are important only for the scattering processes. The imaginary part of diagonal polarization operators for in these regions are presented in Tab. 3. Real parts of the polarization operators are analyzed in Appendix A.

Now we study in detail each of the matrix elements of $\hat{V}$. Following Eq. (A17) the imaginary part of the potential $V_{11}$ for scattering processes and for small arguments $\omega<<\Delta$ and $D q^{2}<<\Delta$ is

$$
\operatorname{Im} V_{11}^{A}(Q)=-\operatorname{Im} \frac{1}{2 / \lambda+\Pi_{11}^{A}(Q)}
$$




$$
\approx \operatorname{Im} \frac{1}{\nu / 2-i \operatorname{Im} \Pi_{11}^{A}(Q)} \approx\left(\frac{1}{\nu}\right)^{2} \operatorname{Im} \Pi_{11}^{A}(Q)
$$

This approximation is justified because there is no singularity in the order parameter amplitude propagator $V_{11}$ at small frequency and momentum for any finite $\Delta$, which means that fluctuations of the amplitude of the order-parameter are massive. For the recombination processes, large frequencies $\omega>2 \Delta$ are important, and according to Tab. $3 \operatorname{Im}_{11}$ may be neglected.

As was shown in Appendix A, the propagators $V_{33}$ and $V_{22}$ for $\omega<<\Delta$ and $D q^{2}<<\Delta$ have the form

$$
\begin{gathered}
V_{33}^{A}(Q)=\frac{\kappa}{\nu q} \frac{\pi \Delta D q^{2}-\omega^{2}}{\pi \Delta D q \kappa-(\omega-i 0)^{2}} \\
V_{22}^{A}(Q)=\frac{\kappa}{\nu q} \frac{4 \Delta^{2}}{\pi \Delta D q \kappa-(\omega-i 0)^{2}} \frac{\pi \Delta D q^{2}-\omega^{2}}{\pi \Delta q^{2}+\omega^{2}} .
\end{gathered}
$$

Thus the imaginary part of the propagators $V_{33}$ and $V_{22}$ for small arguments comes from the pole corresponding to the phase mode, not from the imaginary part of the polarization operators.

For large frequencies $\omega \sim 2 \Delta$ we use the following approximation,

$$
\begin{aligned}
& \operatorname{Im} V_{33}^{A}(Q)=\left(V_{0}^{-1}(q)-\Pi_{33}^{A}(Q)-\frac{\left(\Pi_{23}^{3}(Q)\right)^{2}}{2 / \lambda+\Pi_{22}^{3}(Q)}\right)^{-1} \approx V_{S}(q)^{2} \operatorname{Im} \Pi_{33}^{A}(Q), \\
& \operatorname{Im} V_{22}^{A}(Q)=\operatorname{Im}\left(-\frac{2}{\lambda}-\Pi_{22}^{A}(Q)+\frac{\left(\Pi_{23}^{A}(Q)\right)^{2}}{V_{0}(q)^{-1}-\Pi_{33}^{A}(Q)}\right)^{-1} \approx \frac{\lambda^{2}}{4} \operatorname{Im} \Pi_{22}^{A}(Q) .
\end{aligned}
$$

where $V_{S}(q)=\kappa / \nu(q+\kappa)$ is the statically screened Coulomb potential in the normal state, which was presented above for the two-dimensional case, $\kappa=2 \pi \nu e^{2}$ is the screening momentum. Such an approximation is justified due to absence of collective excitation in this frequency region. 


\section{ELECTRON-ELECTRON RELAXATION}

The kinetic equation for nonequilibrium distribution function in a spatially uniform system is

$$
\frac{d n(\epsilon)}{d t}=-\frac{i}{\pi \nu} \frac{\xi_{\epsilon}}{\epsilon} \frac{1}{2} \operatorname{Tr} \int \frac{d^{2} p}{(2 \pi)^{2}} \operatorname{Im}\left[\hat{G}^{A}(P)\right]\left[\hat{\Sigma}^{C}(P)-S(\epsilon)\left(\hat{\Sigma}^{A}(P)-\hat{\Sigma}^{R}(P)\right)\right]
$$

The electron energy relaxation time $\tau_{e-e}$ is determined from the equation

$$
\frac{1}{\tau_{e-e}(T, \epsilon)}=-\frac{\partial}{\partial n(\epsilon)} \frac{d n(\epsilon)}{d t}
$$

The electron self-energy is shown in Fig. 3. Using the results of Section 2, we have

$$
\begin{array}{r}
\frac{1}{\tau_{e-e}(T, \epsilon)}=\frac{2}{\pi} \frac{\xi_{\epsilon}}{\epsilon} \int \frac{d Q}{(2 \pi)^{3}}[N(\omega)+n(\omega+\epsilon)] \\
\times \frac{\delta}{\delta S(\epsilon)}\left\{\operatorname{Im} V_{i i}^{A}(Q) \operatorname{Re}\left[\operatorname{Pr}_{\hat{\tau}_{i}}\left(\hat{\Gamma}_{11}^{2}\left(\hat{\tau}_{i}\right)\right)\right]-2 \operatorname{Im} V_{23}^{A} \operatorname{Im}\left[\operatorname{Pr}_{\hat{\tau}_{3}}\left(\hat{\Gamma}_{11}^{2}\left(\hat{\tau}_{2}\right)\right)\right]\right\},
\end{array}
$$

where $\operatorname{Pr}_{\hat{\tau}_{i}}$ means the component proportional to matrix $\hat{\tau}_{i}$ (projection on $\hat{\tau}_{i}$ ). In Eq.

summation on repeated indices is implied. Using Tab. 2 and relation $\delta \Gamma_{11}^{2} / \delta S(\epsilon)=\Gamma_{21}^{2}-$ $\left(\Gamma_{22}^{1}\right)^{*}$ we present Eq. (37) in the form

$$
\begin{array}{r}
\frac{1}{\tau_{e-e}(T, \epsilon)}=\frac{1}{\pi^{2}} \int d \omega \int \frac{d^{2} q}{(2 \pi)^{2}}[N(\omega)+n(\omega+\epsilon)] \\
\times\left[\operatorname { I m } V _ { 1 1 } ^ { A } ( Q ) \left(\left(\frac{\xi_{\epsilon}}{\epsilon}+\frac{\epsilon(\epsilon+\omega)+\Delta^{2}}{\epsilon \xi_{\epsilon+\omega}}\right) \frac{D q^{2}}{\left(\xi_{\epsilon+\omega}+\xi_{\epsilon}\right)^{2}+\left(D q^{2}\right)^{2}}\right.\right. \\
\left.-\left(\frac{\xi_{\epsilon}}{\epsilon}-\frac{\epsilon(\epsilon+\omega)+\Delta^{2}}{\epsilon \xi_{\epsilon+\omega}}\right) \frac{D q^{2}}{\left(\xi_{\epsilon+\omega}-\xi_{\epsilon}\right)^{2}+\left(D q^{2}\right)^{2}}\right) \\
+\operatorname{Im} V_{22}^{A}(Q)\left(\left(\frac{\xi_{\epsilon}}{\epsilon}+\frac{\epsilon(\epsilon+\omega)-\Delta^{2}}{\epsilon \xi_{\epsilon+\omega}}\right) \frac{D q^{2}}{\left(\xi_{\epsilon+\omega}+\xi_{\epsilon}\right)^{2}+\left(D q^{2}\right)^{2}}\right. \\
\left.-\left(\frac{\xi_{\epsilon}}{\epsilon}-\frac{\epsilon(\epsilon+\omega)-\Delta^{2}}{\epsilon q_{\epsilon+\omega}}\right) \frac{D q^{2}}{\left(\xi_{\epsilon+\omega}-\xi_{\epsilon}\right)^{2}+\left(D q^{2}\right)^{2}}\right) \\
+\operatorname{Im} V_{33}^{A}(Q)\left(\left(\frac{\xi_{\epsilon}}{\epsilon}-\frac{\epsilon(\epsilon+\omega)-\Delta^{2}}{\epsilon \xi_{\epsilon+\omega}}\right) \frac{D q^{2}}{\left(\xi_{\epsilon+\omega}+\xi_{\epsilon}\right)^{2}+\left(D q^{2}\right)^{2}}\right. \\
\left.-\left(\frac{\xi_{\epsilon}}{\epsilon}+\frac{\epsilon(\epsilon+\omega)-\Delta^{2}}{\epsilon \xi_{\epsilon+\omega}}\right) \frac{D q^{2}}{\left(\xi_{\epsilon+\omega}-\xi_{\epsilon}\right)^{2}+\left(D q^{2}\right)^{2}}\right) \\
\left.+2 i \operatorname{Im} V_{23}^{A} \frac{\omega \Delta}{\epsilon \xi_{\epsilon+\omega}}\left(\frac{D q^{2}}{\left(\xi_{\epsilon+\omega}+\xi_{\epsilon}\right)^{2}+\left(D q^{2}\right)^{2}}+\frac{q^{2}}{\left(\xi_{\epsilon+\omega}-\xi_{\epsilon}\right)^{2}+\left(D q^{2}\right)^{2}}\right)\right] .
\end{array}
$$

In order to separate out the processes of scattering and recombination of quasiparticles we need to make a transformation from the electronic representation to the quasiparticle 
representation in Eq. (38). Note that the presence of imaginary factor $i$ in the last term in Eq. (38) means that for the contribution of the nondiagonal channels of interaction requires the states under the gap to be taken into account according to the equation: $\zeta_{\epsilon+\omega}=$ $i\left[\Delta^{2}-(\omega+\epsilon)^{2}\right]^{1 / 2}, \quad|\epsilon+\omega|<\Delta$. Such states should also be included in equations for the polarization operator $\Pi_{23}$, in Eqs. (28) and (29) only the states above the gap were included. The analysis similar to that presented in Ref. 6 for the clean case shows that contribution from the nondiagonal channels of interaction may be neglected.

For electrons on the Fermi surface, $\epsilon=\Delta$,

$$
\begin{array}{r}
\frac{1}{\tau_{e-e}(T, \epsilon=\Delta)_{\text {scatt }}}=\frac{2}{\pi^{3}} \int_{2 \Delta}^{\infty} d \omega \int_{0}^{\infty} d q q[N(\omega)+n(\omega-\Delta)] \frac{D q^{2}}{\omega^{2}-\Delta \omega+\left(D q^{2}\right)^{2}} \\
\quad \times\left[\left[\operatorname{Im} V_{22}^{A}(Q)_{\text {recom }}+\operatorname{Im} V_{33}^{A}(Q)_{\text {recom }}\right]\left(\frac{\Delta}{2 \omega}\right)^{1 / 2}\right] \\
+\frac{2}{\pi^{3}} \int_{0}^{\infty} d \omega \int_{0}^{\infty} d q q[N(\omega)+n(\omega+\Delta)] \frac{D q^{2}}{2 \Delta \omega+\omega^{2}+\left(D q^{2}\right)^{2}} \\
\times\left[\operatorname{Im} V_{11}^{A}(Q)_{\text {scatt }}\left(\frac{2 \Delta+\omega}{\omega}\right)^{1 / 2}+\left[\operatorname{Im} V_{22}^{A}(Q)_{\text {scatt }}+\operatorname{Im} V_{33}^{A}(Q)_{\text {scatt }}\right]\left(\frac{\Delta}{2 \omega}\right)^{1 / 2}\right] \\
\frac{1}{\tau_{e-e}(T, \epsilon=\Delta)_{\text {recom }}}=\frac{2}{\pi^{3}} \int_{2 \Delta}^{\infty} d \omega \int_{0}^{\infty} d q q[N(\omega)+n(\omega-\Delta)] \frac{D q^{2}}{\omega^{2}-\Delta \omega+\left(D q^{2}\right)^{2}} \\
\times\left[\operatorname{Im} V_{11}^{A}(Q)_{\text {scatt }}\left(\frac{\omega-2 \Delta}{\omega}\right)^{1 / 2}+\left[\operatorname{Im} V_{22}^{A}(Q)_{\text {scatt }}+\operatorname{Im} V_{33}^{A}(Q)_{\text {scatt }}\right]\left(\frac{\Delta}{2 \omega}\right)^{1 / 2}\right] \\
+\frac{2}{\pi^{3}} \int_{2 \Delta}^{\infty} d \omega \int_{0}^{\infty} d q q[N(\omega)+n(\omega+\Delta)] \frac{D q^{2}}{2 \Delta \omega+\omega^{2}+\left(D q^{2}\right)^{2}} \\
\left.\times\left[\operatorname{Im} V_{22}^{A}(Q)_{\text {recom }}+\operatorname{Im} V_{33}^{A}(Q)_{\text {recom }}\right]\left(\frac{\Delta}{2 \omega}\right)^{1 / 2}\right] .
\end{array}
$$

Eqs. (39) and (40) describe processes of scattering "two into two" and "three into one" quasiparticles correspondingly.

Further calculations will be performed for low temperatures $T<<\Delta$. It may be shown that the most important contribution to the recombination time originates from terms $V_{22}(Q)_{\text {recom }}$ and $V_{33}(Q)_{\text {recom }}$ in Eq. (40),

$$
\frac{1}{\tau_{e-e}(T, \epsilon=\Delta)_{\text {recom }}}=\frac{T}{4 \pi D \nu}\left(1+\left(\frac{\lambda \nu}{2}\right)^{2}\right) \exp \left(-\frac{2 \Delta}{T}\right)
$$

Calculating the scattering relaxation time from terms $V_{22}(Q)_{\text {recom }}$ and $V_{33}(Q)_{\text {recom }}$ in Eq. (38) we use the approximation of Eqs. (33) and (34) and we use Tab. 3 for the imaginary parts of the polarization operators. As a result we get 


$$
{\frac{1}{\tau_{e-e}(T, \epsilon=\Delta)_{s c a t t}}}=\frac{T}{2 \pi^{2} D \nu}\left(1+\left(\frac{\lambda \nu}{2}\right)^{2}\right) \exp \left(-\frac{\Delta}{T}\right) .
$$

As for the contribution to the scattering relaxation time from terms $\operatorname{Im} V_{22}(Q)_{\text {scatt }}$ and $\operatorname{Im} V_{33}(Q)_{\text {scatt }}$ in Eq. (38) we note that for small energy transfers, $\omega<<T<<\Delta$ the imaginary part of the propagators $V_{33}$ and $V_{22}$ originates from the poles corresponding to the phase mode as seen in Eqs. (31) and (32)). Integrating these poles over the momentum $q$ we get

$$
{\frac{1}{\tau_{e-e}(T, \epsilon=\Delta)_{s c a t t}}}=\frac{21}{2 \pi^{6}}\left(\frac{\pi}{2}\right)^{1 / 2}\left(\frac{T}{\Delta}\right)^{3 / 2} \frac{T^{2}}{D^{2} \kappa^{2} \nu} .
$$

Note that the main contribution comes from the propagator $V_{22}$ corresponding to the fluctuation of the phase of the order parameter.

The term $V_{11}(Q)_{\text {scatt }}$ does not have poles corresponding to the collective mode, thus using Eq. (30) we find that the scattering relaxation time has a power law divergence,

$$
\frac{1}{\tau_{e-e}(T, \epsilon=\Delta)_{s c a t t}}=\frac{\Delta}{2 \pi^{2} D \nu}\left(\frac{\pi T}{\omega_{0}}\right)^{1 / 2} \exp \left(-\frac{\Delta}{T}\right)
$$

This divergence is similar to the logarithmic divergence of the phase or energy relaxation times in the normal impure two-dimensional case ${ }^{14}$. According to Ref. 14 the cutoff frequency $\omega_{0}$ is defined by the relaxation time $\tau_{e-e}$, which physically means that the kinetic equation cannot be applied for energy transfers less than $1 / \tau_{e-e}$, thus the self-consistent solution of Eq. (44) is

$$
{\frac{1}{\tau_{e-e}(T, \epsilon=\Delta)}}_{s c a t t}=\frac{\left(\pi \Delta^{2} T\right)^{1 / 3}}{\left(2 \pi^{2} D \nu\right)^{3 / 2}} \exp \left(-\frac{2 \Delta}{3 T}\right) .
$$

The low-frequency singularity in the scattering relaxation time mentioned above is for electrons exactly at the Fermi surface, $\epsilon=\Delta$. We note that for the electrons above the Fermi surface, $\epsilon>\Delta$ the singularity in the scattering relaxation time associated with the potential $V_{11}(Q)_{\text {scatt }}$ is weaker but it does not disappear. More accurately such a divergence must be regularized directly in the physically measurable quantity e.g. the tunneling conductance. However it is not necessary because the contribution to the scattering relaxation time from 
the phase collective mode, Eq. (43), is more important because it does not have a small exponential factor such as that presented in Eqs. (42) and (45).

The appearance of the nonexponential scattering relaxation at low temperature is a direct consequence of the gapless phase mode in two dimensions. In three dimensions the phase mode have a gap and the main contribution to the scattering relaxation comes from the potential $V_{11}$.

$$
{\frac{1}{\tau_{e-e}(T, \epsilon=\Delta)_{s c a t t}}}=\frac{12 \Delta T^{1 / 2}}{\pi(\pi D)^{3 / 2} \nu_{3}}\left(\frac{\Delta}{\omega_{0}}\right)^{1 / 4} \exp \left(-\frac{\Delta}{T}\right)
$$

where $\nu_{3}=m p_{F} / \pi^{2}$ is the three-dimensional density of states. Again after regularization of singularity in Eq. (46) we have

$$
{\frac{1}{\tau_{e-e}(T, \epsilon=\Delta)_{s c a t t}}}_{=}=\frac{12 T^{1 / 2}}{\pi(\pi D)^{3 / 2} \nu_{3}} \Delta \exp \left(-\frac{4 \Delta}{5 T}\right) .
$$

The recombination relaxation time is obtained similar to Eq. (41),

$$
\frac{1}{\tau_{e-e}(T, \epsilon=\Delta)_{\text {recom }}}=\frac{1}{2^{1 / 4}(2 \pi)^{2}} \frac{\Delta^{1 / 2} T}{D^{3 / 2} \nu_{3}}\left(1+\left(\frac{\lambda \nu_{3}}{2}\right)^{2}\right) \exp \left(-\frac{2 \Delta}{T}\right)
$$

\section{TWO-LAYER SUPERCONDUCTOR-NORMAL METAL SYSTEM}

We consider a system of two disordered electron layers with different density of states, $\nu_{1,2}$, elastic scattering times, $\tau_{1,2}$, mean free paths, $\ell_{1,2}$, and diffusion coefficients $D_{1,2}$. The layers are coupled by the Coulomb potentials, there is no superconducting coupling between the layers.

First we consider the screened Coulomb potentials in the normal state. The nonscreened Coulomb potentials within the layer, $V_{0}$, and between electrons in different planes, $U_{0}$, are

$$
V_{0}(q)=2 \pi e^{2} / q \epsilon, \quad U_{0}(q)=\frac{1}{\epsilon_{1}} \frac{2 \pi e^{2}}{q} \exp (-q b)
$$

where $\epsilon$ and $\epsilon_{1}$ are the dielectric constants of the electron layer and the inter-layer media, $b$ is the distance between layers. We assume that $\epsilon \approx \epsilon_{1}$ and we absorbed $\epsilon$ into $e^{2}$ 
In all further calculations small momentum transfers are important, thus we assume $q b<<1$

$$
V_{0}-U_{0}=V_{0}(1-\exp (-q b))=2 \pi e^{2} b, \quad V_{0}^{2}-U_{0}^{2}=V_{0} 4 \pi e^{2} b .
$$

In this chapter the lower indices of the potentials and the polarization operators refer to the layer, e.g. $V_{11}$ means the Coulomb potential between electrons in the layer 1 , etc. The screened potentials are satisfied the equations

$$
\left(\begin{array}{ll}
V_{11} & U_{12} \\
U_{21} & V_{22}
\end{array}\right)=\left(\begin{array}{ll}
V_{0} & U_{0} \\
U_{0} & V_{0}
\end{array}\right)+\left(\begin{array}{cc}
V_{0} & U_{0} \\
U_{0} & V_{0}
\end{array}\right)\left(\begin{array}{cc}
\Pi_{1} & 0 \\
0 & \Pi_{2}
\end{array}\right)\left(\begin{array}{cc}
V_{11} & U_{12} \\
U_{21} & V_{22}
\end{array}\right) .
$$

We will use the definitions: $V_{11}=V_{1}, V_{22}=V_{2}$, and $U_{12}=U_{21}=U$. The solution of Eq. (3) is

$$
\begin{array}{r}
U=\frac{U_{0}}{P}, \quad V_{1}=\frac{V_{0}-\left(V_{0}^{2}-U_{0}^{2}\right) \Pi_{2}}{P}, \quad V_{2}=\frac{V_{0}-\left(V_{0}^{2}-U_{0}^{2}\right) \Pi_{1}}{P}, \\
P=\left(1-V_{0} \Pi_{1}\right)\left(1-V_{0} \Pi_{2}\right)-U_{0}^{2} \Pi_{1} \Pi_{2} \approx 1-V_{0}\left(\Pi_{1}+\Pi_{2}-4 \pi e^{2} b \Pi_{1} \Pi_{2}\right) .
\end{array}
$$

The polarization operators in each layers for $q \ell_{i}<<1$ and $\omega \tau_{i}<<1$ are chosen in the form corresponding to a normal state, because for recombination processes large frequencies $\omega>$ $2 \Delta$ are important, while collective excitations in a superconductor exist only for $\omega<<\Delta$.

$$
\Pi_{i}^{A}(Q)=-\nu_{i} \frac{D_{i} q^{2}}{i \omega+D_{i} q^{2}}
$$

The potentials are

$$
\begin{gathered}
U^{A}(Q)=\frac{1}{\nu_{1}\left(D_{1}+D_{2} \nu_{2} / \nu_{1}\right) q^{2}} \frac{\left(i \omega+D_{1} q^{2}\right)\left(i \omega+D_{2} q^{2}\right)}{i \omega+\tilde{D} q^{2}} \\
V_{1}^{A}(Q)=U^{A}(Q) \frac{i \omega+\left(1+2 \kappa_{2} d\right) D_{2} q^{2}}{i \omega+D_{2} q^{2}}
\end{gathered}
$$

where

$$
\tilde{D}=\left(1+\frac{\nu_{2}}{\nu_{1}}+2 \kappa_{2} d\right) \frac{D_{1} D_{2}}{D_{1}+D_{2} \nu_{2} / \nu_{1}}, \quad \kappa_{2}=2 \pi e^{2} \nu_{2}
$$


We assume that layer 1 is in the superconducting state and layer 2 is in the normal state. We will calculate the recombination relaxation time in the superconducting layer due to inter-layer electron-electron interaction $U$. From Eq. (40) we have

$$
\begin{aligned}
\frac{1}{\tau_{e-e}(T, \epsilon=\Delta)_{\text {recom }}}=\frac{2}{\pi^{3}} \int_{2 \Delta}^{\infty} d \omega \int_{0}^{\infty} d q q[N(\omega)+n(\omega-\Delta)] & \frac{D_{1} q^{2}}{\omega^{2}-\Delta \omega+\left(D_{1} q^{2}\right)^{2}} \\
& \operatorname{Im} U^{A}(Q)\left(\frac{\Delta}{2 \omega}\right)^{1 / 2}
\end{aligned}
$$

For the imaginary part of the iner-layer interaction we use the approximation similar to Eq. (33), $\operatorname{Im} U^{A}(q, \omega)=|U(q, 0)|^{2} \operatorname{Im} \Pi_{2}^{A}(Q)$, where $U(q, 0)$ corresponds to the static limit of Eq. (53). The imaginary part of the polarization operator $\Pi_{2}$ corresponds to the electron scattering in the normal layer and thus does not have a small exponential factor typical for a superconductor. As a result

$$
\frac{1}{\tau_{e-e}(T, \epsilon=\Delta)_{\text {recom }}}=\frac{T}{4 \pi^{2} \nu_{1}} \frac{D_{1} D_{2}}{\left(D_{1}+D_{2} \nu_{2} / \nu_{1}\right)^{2}}\left(\frac{1}{\tilde{D}}+\frac{1}{\tilde{D}+\sqrt{2} D_{1}}\right) \exp \left(-\frac{\Delta}{T}\right) .
$$

We see that relaxation rate in a superconductor-normal metal two-layer system is increased by an exponential factor in comparison with the recombination rate in a single layer, see Eq. (40).

\section{CONCLUSIONS}

We derived the kinetic equation describing the electron relaxation in two-dimensional impure superconductors. For electrons at the Fermi surface, $\epsilon=\Delta$, the recombination and scattering relaxation time were calculated for low temperatures, $T<<\Delta$. We took into account all channels of the electron-electron interaction in the superconductor.

We found that the recombination relaxation rate comes from the quasiparticle scattering (recombination processes) associated with the fluctuations of the electron density and the phase of the order parameter, the propagators $V_{33}$ and $V_{22}$. The recombination relaxation rate has double exponential smallness at low temperatures (see Eq. (41)), associated with exponentially small number of available quasiparticles. 
The scattering relaxation rate has a power law temperature dependence (see Eq. (43)) due to singularity in the propagators $V_{22}$ and $V_{33}$ associated with the gapless collective mode, the phase mode. The contribution to the scattering relaxation rate from the fluctuations of the amplitude of the order parameter, $V_{11}$ has an infrared divergence similar to the phase relaxation time in the two-dimensional normal metal [14], however after regularization the corresponding contribution to the scattering relaxation rate has a small exponential factor and therefore is less important that the contribution from the collective excitations.

We also shown that in the superconductor-normal metal two-layer system the recombination relaxation rate in the superconducting layer due to the inter-layer Coulomb interaction is strongly increased at low temperatures $T<<\Delta$ by an exponential factor $\exp (\Delta / T)>>1$ in comparison with a single superconducting layer. this fact may be important for constructing superconducting radiation detectors [2] .

The author is grateful to I. L. Aleiner for valuable discussions and A. V. Sergeev for his help at the early stage of the work.

\section{APPENDIX A:}

In this Appendix we obtain equations for the polarization operators for some limiting cases and prove some identities for them. Though some of the results were already presented in Ref. 8, for Matsubara frequencies and in Ref. 15 for $T=0$ the analysis for continious frequencies has some advantages and helps us to estimate the imaginary parts of the interaction propagators.

We start with the polarization operators for $q=0$. $\Pi_{23}(0, \omega)$ may be taken directly from Eq. (21),

$$
\Pi_{23}(0, \omega)=-\frac{i \nu \omega \Delta}{4} J(\omega)
$$

where 


$$
J(\omega)=\int \frac{d \epsilon}{\xi_{\epsilon} \xi_{\epsilon+\omega}}\left(\frac{S(\epsilon+\omega)-S(\epsilon)}{\xi_{\epsilon+\omega}-\xi_{\epsilon}}-\frac{S(\epsilon+\omega)+S(\epsilon)}{\xi_{\epsilon+\omega}+\xi_{\epsilon}}\right) .
$$

Then we take $\Pi_{22}(\omega, 0)$ from Eq. (19),

$$
\begin{aligned}
\Pi_{22}(0, \omega)=\frac{\nu}{4} \int d \epsilon[ & \left(1-\frac{\epsilon(\epsilon+\omega)-\Delta^{2}}{\xi_{\epsilon} \xi_{\epsilon+\omega}}\right) \frac{S(\epsilon+\omega)-S(\epsilon)}{\xi_{\epsilon+\omega}-\xi_{\epsilon}}+ \\
& \left.\left(1+\frac{\epsilon(\epsilon+\omega)-\Delta^{2}}{\xi_{\epsilon} \xi_{\epsilon+\omega}}\right) \frac{S(\epsilon+\omega)+S(\epsilon)}{\xi_{\epsilon+\omega}+\xi_{\epsilon}}\right],
\end{aligned}
$$

and transform it using the identities

$$
\begin{aligned}
& 1-\frac{\epsilon(\epsilon+\omega)-\Delta^{2}}{\xi_{\epsilon} \xi_{\epsilon+\omega}}=-\frac{\left(\xi_{\epsilon+\omega}-\xi_{\epsilon}\right)^{2}-\omega^{2}}{2 \xi_{\epsilon} \xi_{\epsilon+\omega}}, \\
& 1+\frac{\epsilon(\epsilon+\omega)-\Delta^{2}}{\xi_{\epsilon} \xi_{\epsilon+\omega}}=-\frac{\left(\xi_{\epsilon+\omega}+\xi_{\epsilon}\right)^{2}-\omega^{2}}{2 \xi_{\epsilon} \xi_{\epsilon+\omega}},
\end{aligned}
$$

to the form

$$
\Pi_{22}(0, \omega)=\frac{\nu}{4} \int d \epsilon\left(\frac{S(\epsilon)}{\xi_{\epsilon}}+\frac{S(\epsilon+\omega)}{\xi_{\epsilon+\omega}}\right)+\frac{\nu \omega^{2}}{8} J(\omega) .
$$

Now recalling the BCS selfconsistancy equation

$$
\frac{2}{\lambda}+\frac{\nu}{2} \int d \epsilon \frac{S(\epsilon)}{\xi_{\epsilon}}=0
$$

we see that

$$
\frac{2}{\lambda}+\Pi_{22}(0, \omega)=\frac{\nu \omega^{2}}{8} J(\omega)
$$

To transform $\Pi_{33}(0, \omega)$,

$$
\begin{aligned}
\Pi_{33}(0, \omega)+\nu=-\frac{\nu}{4} \int d \epsilon[ & \left(1+\frac{\epsilon(\epsilon+\omega)-\Delta^{2}}{\xi_{\epsilon} \xi_{\epsilon+\omega}}\right) \frac{S(\epsilon+\omega)-S(\epsilon)}{\xi_{\epsilon+\omega}-\xi_{\epsilon}}+ \\
& \left.\left(1-\frac{\epsilon(\epsilon+\omega)-\Delta^{2}}{\xi_{\epsilon} \xi_{\epsilon+\omega}}\right) \frac{S(\epsilon+\omega)+S(\epsilon)}{\xi_{\epsilon+\omega}+\xi_{\epsilon}}\right],
\end{aligned}
$$

we use the identities

$$
\begin{aligned}
& 1+\frac{\epsilon(\epsilon+\omega)-\Delta^{2}}{\xi_{\epsilon} \xi_{\epsilon+\omega}}=\frac{\xi_{\epsilon+\omega}-\xi_{\epsilon}}{\omega}\left(\frac{\epsilon+\omega}{\xi_{\epsilon+\omega}}+\frac{\epsilon}{\xi_{\epsilon}}\right)-\frac{2 \Delta^{2}}{\xi_{\epsilon} \xi_{\epsilon+\omega}}, \\
& 1-\frac{\epsilon(\epsilon+\omega)-\Delta^{2}}{\xi_{\epsilon} \xi_{\epsilon+\omega}}=\frac{\xi_{\epsilon+\omega}+\xi_{\epsilon}}{\omega}\left(\frac{\epsilon+\omega}{\xi_{\epsilon+\omega}}-\frac{\epsilon}{\xi_{\epsilon}}\right)+\frac{2 \Delta^{2}}{\xi_{\epsilon} \xi_{\epsilon+\omega}},
\end{aligned}
$$


and get

$$
\Pi_{33}(0, \omega)+\nu=-\frac{\nu}{2 \omega} \int d \epsilon\left(\frac{\epsilon+\omega}{\xi_{\epsilon+\omega}} S(\epsilon+\omega)-\frac{\epsilon}{\xi_{\epsilon}} S(\epsilon)\right)+\frac{\nu \Delta^{2}}{2} J(\omega) .
$$

Now we recall another identity,

$$
\int d \epsilon\left(\frac{\epsilon+\omega}{\xi_{\epsilon+\omega}} S(\epsilon+\omega)-\frac{\epsilon}{\xi_{\epsilon}} S(\epsilon)\right)=-2 \omega,
$$

and get

$$
\Pi_{33}(0, \omega)=\frac{\nu \Delta^{2}}{2} J(\omega)
$$

We see from Eqs. (A5), (A8), and (A13) that the following identity holds

$$
\Pi_{33}(0, \omega)\left(\frac{2}{\lambda}+\Pi_{22}(0, \omega)\right)+\left(\Pi_{23}(0, \omega)\right)^{2}=0 .
$$

At low temperatures, $T<<\Delta$ and for small arguments $\omega<<\Delta$ and $D q^{2}<<\Delta$, the polarization operators are

$$
\begin{gathered}
\frac{2}{\lambda}+\Pi_{11}(Q)=-\nu\left[1-\frac{\omega^{2}}{12 \Delta^{2}}+\pi \frac{D q^{2}}{8 \Delta^{2}}\right], \\
\frac{2}{\lambda}+\Pi_{22}(Q)=-\nu\left[-\frac{\omega^{2}}{4 \Delta^{2}}+\pi \frac{D q^{2}}{4 \Delta^{2}}\right], \\
\Pi_{23}(Q)=-i \nu \frac{\omega}{2 \Delta}, \quad \Pi_{33}(Q)=-\nu\left[1+\frac{\omega^{2}}{6 \Delta^{2}}\right] .
\end{gathered}
$$

Thus for small arguments the following relation holds [ [ ]

$$
\Pi_{33}(Q)\left(\frac{2}{\lambda}+\Pi_{22}(Q)\right)+\left(\Pi_{23}(Q)\right)^{2} \sim q^{2} .
$$

Using Eq. (A16) we have for the propagator $V_{11}$

$$
V_{11}(Q)=-\left(2 / \lambda+\Pi_{11}(Q)\right)^{-1}=\nu\left(1-\frac{\omega^{2}}{12 \Delta^{2}}+\frac{\pi}{8} \frac{D q^{2}}{\Delta^{2}}\right)^{-1} .
$$

We see that $V_{11}$ is not singular which means that fluctuations of the amplitude of the order parameter are massive, thus the imaginary part of the propagator $V_{11}$ originates from $\operatorname{Im} \Pi_{11}(Q)$. 
The screened Coulomb potential is presented in the form

$$
V_{33}^{A}(Q)=\frac{V_{0}(q)}{1-V_{0}(q) \tilde{\Pi}^{A}(Q)},
$$

where

$$
\tilde{\Pi}^{A}(Q)=\Pi_{33}^{A}(q, \omega)+\frac{\left(\Pi_{23}^{A}(Q)\right)^{2}}{2 / \lambda+\Pi_{22}^{A}(Q)}=-\nu \frac{\pi \Delta D q^{2}}{\pi \Delta D q^{2}-(\omega-i 0)^{2}}
$$

The propagator $V_{22}$ may be written in a similar way

$$
V_{22}^{A}(Q)=-\frac{1}{\frac{2}{\lambda}+\Pi_{22}^{A}(Q)} \frac{1-V_{0}(q) \Pi_{33}^{A}(Q)}{1-V_{0}(q) \tilde{\Pi}^{A}(Q)} .
$$

The poles in the propagators $V_{33}$ and $V_{22}$ correspond to the collective excitation, the phase mode, which in two dimensions is 15 given by equation $\omega^{2}=\pi \Delta D \kappa q$.

In quasi-one-dimensional superconductors the nonscreened Coulomb potential is $V_{0}(q)=$ $2 e^{2} \ln (1 / q a), q a<<1$, where $a$ is a cross-sectional size, and the density of states is $\nu_{1}=$ $1 / \pi v_{F}$, thus the spectrum of the pase mode is [18]

$$
\omega^{2}=\pi \Delta D q^{2}\left(\frac{2 e^{2}}{\pi v_{F}} \ln (1 / q a)-1\right)
$$

\section{APPENDIX B:}

It is interesting to see how the spectrum of the phase mode changes in different two-layer systems. First we consider a system of two identical impure superconducting planes coupled by the Coulomb interaction, but no Josephson coupling between the planes, thus the order parameters are independent in each planes. For the screened Coulomb potential in each layer we have from Eq. (52)

$$
V(Q)=\frac{V_{0}(q)-\left[V_{0}^{2}(q)-U_{0}^{2}(q)\right] \tilde{\Pi}(Q)}{\left[1-V_{0}(q) \tilde{\Pi}(Q)\right]^{2}-\left[U_{0}(q) \tilde{\Pi}(Q)\right]^{2}} .
$$

To avoid confusion we dropped lower indices in the Coulomb potential $V$. The spectrum of the phase modes is defined by equations $1-\left(V_{0}+U_{0}\right) \tilde{\Pi}=0$ and $1-\left(V_{0}-U_{0}\right) \tilde{\Pi}=0$. the solution of these equations for $q d<<1$ is $\omega_{+}=(2 \pi \Delta D \kappa q)^{1 / 2}$ and $\omega_{-}=(2 \pi \Delta D \kappa d)^{1 / 2} q$. 
These new phase modes are similar to in-phase and out-of-phase plasmons in symmetric two-layer clean normal metal system, see [16] and [17].

In a system of two coupled quasi-one-dimensional disordered superconductors the spectrum of the phase modes in the long-wave limit $q d<<1$ is

$$
\omega_{+}^{2}=\pi \Delta D q^{2}\left(\frac{4 e^{2}}{\pi v_{F}} \ln (1 / q a)-1\right), \quad \omega_{-}^{2}=\pi \Delta D q^{2}\left(\frac{2 e^{2}}{\pi v_{F}} \ln (d / a)-1\right) .
$$

Now we consider a two-layer superconductor-normal metal disordered system. The polarization operators in the superconducting and normal layers according to Eqs. (A22) and (53) are

$$
\begin{gathered}
\Pi_{1}^{A}(Q)=-\nu_{s} \frac{\pi \Delta D_{s} q^{2}}{\pi \Delta D_{s} q^{2}-(\omega-i 0)^{2}}, \\
\Pi_{2}^{A}(Q)=-\nu_{n} \frac{\pi D_{n} q^{2}}{i \omega+D_{n} q^{2}}
\end{gathered}
$$

The spectrum of collective excitations is determined from the equation

$$
1-V_{0}\left(\Pi_{1}+\Pi_{2}-4 \pi e^{2} b \Pi_{1} \Pi_{2}\right)=0 .
$$

For small momenta $q<<\kappa_{s}, \kappa_{s}=2 \pi e^{2} \nu_{s}$ Eq. (B4) leads to

$$
\omega^{2}=\pi \Delta D_{s} q^{2} A+i \omega \pi \Delta \frac{D_{s}}{D_{n}}, \quad A=1+\frac{\nu_{s}}{\nu_{n}}+2 \kappa_{s} b
$$

The solution of Eq. (B5) is a phase mode with small damping

$$
\begin{array}{r}
\omega= \\
\left(\pi \Delta D_{s} A\right)^{1 / 2} q+\frac{i}{2} \pi \Delta \frac{D_{s}}{D_{n}} \\
\frac{\pi}{A}\left(\frac{D_{s}}{D_{n}}\right)^{2}<<\frac{D_{s} q^{2}}{\Delta}<<1
\end{array}
$$

The last inequality is satisfied provided $A>>1$ and $D_{s}<<D_{n}$. This result was independently obtained in [19]. If the opposite inequality is valid, the solution of Eq. (B6) is a diffusion mode,

$$
i \omega+D_{n} q^{2} A=0, \quad \frac{D_{s} q^{2}}{\Delta}<<\min \left[1, \frac{\pi}{A}\left(\frac{D_{s}}{D_{n}}\right)^{2}\right] .
$$




\section{REFERENCES}

[1] Nonequilibrium Superconductivity, Ed. By D. N. Langenberg and A. I. Larkin, Elsevier (1986).

[2] A. V. Sergeev and M. Reizer, Int. J. Mod. Phys. B 10, 635 (1996).

[3] D. S. Pyun and T. R. Lemberger, Phys. Rev. B 43, 3732 (1991).

[4] S. G. Doettinger, S. Kittenberger, R. P. Huebener, and C. C. Tsuei, Phys. Rev. B 56, 14157 (1997).

[5] M. Yu. Reizer, Phys. Rev. B 39, 1602 (1989).

[6] M. Yu. Reizer, Phys. Rev. B. 57, 1147 (1998).

[7] I. O. Kulik, O. Entin-Wohlman, and R. Orbach, J. Low Temp. Phys. 43, 591 (1981).

[8] R. A. Smith, M. Reizer, J. W. Wilkins, Phys. Rev. B 51, 6470 (1995).

[9] J. R. Shrieffer, Theory of superconductivity, Chapter 8, Addison-Wesley, Redwood City, 1988.

[10] A. Schmid, Z. Phys. 271, 251 (1974).

[11] B. L. Altshuler and A. G. Aronov, Pis'ma Zh. Exsp. Teor. Fiz. 30, 514 (1979); [Sov. Phys. JETP Lett. 30, 482 (1979)]. For a review see B. L. Altshuler and A. G. Aronov, Electron-Electron Interaction in Disordered Systems, edited by A. L. Efros and M. Polak North-Holland, Amsterdam, 1985.

[12] T. P. Devereaux and D. Belitz, Phys. Rev. 44, 4587 (1991); J. Low Temp. Phys. 77, 319 (1989).

[13] M. Yu. Reizer and A. V. Sergeev, Zh. Eksp. Teor. Fiz. 90, 1056 (1986) [Sov. Phys. ZETP 63, 616 (1986)].

[14] B. L. Altshuler, A. G. Aronov, and D. E. Khmel'nitskii, J. Phys. C 15, 7367 (1982). 
[15] U. Eckern and F. Pelzer, J. Low Temp. Phys. 73, 433 (1988).

[16] Y. Takada, J. Phys. Soc. JPN. 43, 1627 (1977);

[17] S. Das Sarma and A. Madhukar, Phys. Rev. B 23, 805 (1981).

[18] J. E. Mooij and G. Schön, Phys. Rev. Lett. 55, 114 (1985).

[19] B. N. Narozhny, I. L. Aleiner, and B. L. Altshuler, Phys. Rev. B (1999). 


\section{FIGURES}

FIG. 1. $\Gamma\left(\hat{\tau}_{i}\right)$ is the impurity renormalized scalar vertex in the ladder approximation, $V_{i j}$ is the screened electron-electron interaction, $\Sigma$ is the electron self-energy in an impure superconductor. 


\section{TABLES}

TABLE I. Summary of the results for $\eta_{i}^{A A}$ and $\eta_{i}^{A R}$ defined by equation Eq. (7). Quantities $A, A_{+}, B$, and $B_{+}$are defined by Eq. (9).

\begin{tabular}{lcc}
\hline \hline$\eta_{i}$ & $A A$ & $A R$ \\
\hline$\eta_{0}$ & $\frac{\zeta_{+}}{2}\left[\left(1-A_{+}\right) \hat{\tau}_{0}-B_{+} \hat{\tau}_{1}\right]$ & $\frac{\zeta}{2}\left[\left(1+A_{+}\right) \hat{\tau}_{0}+B_{+} \hat{\tau}_{1}\right]$ \\
$\eta_{1}$ & $\frac{\zeta_{+}}{2}\left[\left(1+A_{+}\right) \hat{\tau}_{1}+B_{+} \hat{\tau}_{0}\right]$ & $\frac{\zeta}{2}\left[\left(1-A_{+}\right) \hat{\tau}_{1}-B_{+} \hat{\tau}_{0}\right]$ \\
$\eta_{2}$ & $\frac{\zeta_{+}}{2}\left[(1+A) \hat{\tau}_{2}+i B \hat{\tau}_{3}\right]$ & $\frac{\zeta}{2}\left[(1-A) \hat{\tau}_{2}-i B \hat{\tau}_{3}\right]$ \\
$\eta_{3}$ & $\frac{\zeta_{+}}{2}\left[(1-A) \hat{\tau}_{3}+i B \hat{\tau}_{2}\right]$ & $\frac{\zeta}{2}\left[(1+A) \hat{\tau}_{3}-i B \hat{\tau}_{2}\right]$ \\
\hline \hline
\end{tabular}

TABLE II. Summary of the results for impurity renormalized vertices $\Gamma_{22}^{1}\left(\hat{\tau}_{i}\right)$ and $\Gamma_{21}^{2}\left(\hat{\tau}_{i}\right)$.

\begin{tabular}{lcccc}
\hline \hline$\Gamma\left(\hat{\tau}_{i}\right)$ & $\hat{\tau}_{0}$ & $\hat{\tau}_{1}$ & $\hat{\tau}_{2}$ & $\hat{\tau}_{3}$ \\
\hline$\Gamma_{22}^{1}\left(\hat{\tau}_{1}\right)$ & $-\frac{\zeta}{2} \frac{B_{+}}{1-\zeta}$ & $1+\frac{\zeta}{2} \frac{1-A_{+}}{1-\zeta}$ & 0 & 0 \\
$\Gamma_{21}^{2}\left(\hat{\tau}_{1}\right)$ & $\frac{\zeta_{+}}{2} \frac{B_{+}}{1-\zeta_{+}}$ & $1+\frac{\zeta_{+}}{2} \frac{1+A_{+}}{1-\zeta_{+}}$ & 0 & 0 \\
$\Gamma_{22}^{1}\left(\hat{\tau}_{2}\right)$ & 0 & 0 & $1+\frac{\zeta}{2} \frac{1-A}{1-\zeta}$ & $-i \frac{\zeta}{2} \frac{B}{1-\zeta}$ \\
$\Gamma_{21}^{2}\left(\hat{\tau}_{2}\right)$ & 0 & 0 & $1+\frac{\zeta_{+}}{2} \frac{1+A}{1-\zeta_{+}}$ & $i \frac{\zeta_{+}}{2} \frac{B}{1-\zeta_{+}}$ \\
$\Gamma_{22}^{1}\left(\hat{\tau}_{3}\right)$ & 0 & 0 & $-i \frac{\zeta}{2} \frac{B}{1-\zeta}$ & $1+\frac{\zeta}{2} \frac{1+A}{1-\zeta}$ \\
$\Gamma_{21}^{2}\left(\hat{\tau}_{3}\right)$ & 0 & 0 & $i \frac{\zeta_{+}}{2} \frac{B}{1-\zeta_{+}}$ & $1+\frac{\zeta_{+}}{2} \frac{1-A}{1-\zeta_{+}}$ \\
\hline \hline
\end{tabular}

TABLE III. Summary of the results for the polarization operators in the regions of the parameters important for the electron relaxation at low temperatures $T<<\Delta$.

\begin{tabular}{ccc}
\hline \hline $\operatorname{Im} \Pi_{i i}^{A}$ & $\omega-2 \Delta<<T<<\Delta$ & $\omega<<T<<$ \\
\hline$\left(\operatorname{Im} \Pi_{22}^{A}=\operatorname{Im} \Pi_{33}^{A}\right)_{\text {scatt }}$ & $\nu \frac{(\pi \omega T)^{1 / 2} D q^{2} \exp (-\Delta / T)}{\omega^{2}+2 \Delta \omega+\left(D q^{2}\right)^{2}}$ & $2 \nu \frac{\omega D q^{2} \exp (-\Delta / T)}{2 \Delta \omega+\left(D q^{2}\right)^{2}}$ \\
$\left(\operatorname{Im} \Pi_{11}^{A}\right)_{\text {scatt }}$ & $\nu\left(\frac{\pi T}{\omega}\right)^{1 / 2} \frac{(2 \Delta+\omega) D q^{2} \exp (-\Delta / T)}{\omega^{2}+2 \Delta \omega+\left(D q^{2}\right)^{2}}$ & $\nu\left(\frac{\pi \omega}{T}\right)^{1 / 2} \frac{2 \Delta D q^{2} \exp (-\Delta / T)}{2 \Delta \omega+\left(D q^{2}\right)^{2}}$ \\
$\left(\operatorname{Im} \Pi_{22}^{A}=\operatorname{Im} \Pi_{33}^{A}\right)_{\text {rec }}$ & $\nu \frac{\pi \omega D q^{2}}{\omega^{2}-2 \Delta \omega+\left(D q^{2}\right)^{2}}$ & - \\
$\left(\operatorname{Im} \Pi_{11}^{A}\right)_{\text {rec }}$ & $\approx 0$ & - \\
\hline \hline
\end{tabular}




$$
\begin{aligned}
& \Gamma\left(\tau_{i}\right) \varangle=\tau_{i}<+\Gamma\left(\tau_{i}\right)< \\
& v_{i j} W_{\tau_{i}}^{M}=W_{\tau_{i}}^{M}+\underset{\tau_{i}}{m}+W_{\tau_{k}} \\
& \Sigma=\Delta^{\mathrm{i}_{\mathrm{ij}}}
\end{aligned}
$$

